\title{
Use of PET/CT to detect local and regional laryngeal cancer recurrence after surgery
}

This article was published in the following Dove Press journal:

Reports in Medical Imaging

15 June 2017

Number of times this article has been viewed

\author{
Eugenia Allegra' \\ Vincenzo Saita ${ }^{2}$ \\ Massimo De Natale ${ }^{2}$ \\ Nicolò Marino ${ }^{2}$ \\ Serena Trapasso' \\ Stefania Tamburrini ${ }^{3}$ \\ Caterina Alessio 4 \\ Massimo Ippolito 5 \\ 'Otolaryngology, Department of \\ Health Sciences, University of \\ Catanzaro, Catanzaro, ${ }^{2}$ Department \\ of Otolaryngology, Cannizzaro \\ Hospital, Catania, ${ }^{3}$ Department \\ of Radiology, Pellegrini Hospital, \\ Naples, ${ }^{4}$ Department of Experimental \\ and Clinical Medicine-Radiology, \\ University of Catanzaro, Catanzaro, \\ ${ }^{5}$ Department of Nuclear Medicine, \\ Cannizzaro Hospital, Catania, Italy
}

Correspondence: Eugenia Allegra Otolaryngology, Head and Neck Surgery, Department of Health Sciences, University Magna Graecia of Catanzaro, Viale Europa, Germaneto 88I00,

Catanzaro, Italy

Tel +39 96I 3647130

$\mathrm{Fax}+399613647131$

Email eualle@unicz.it
Background: Laryngeal cancer is the second most common cancer of the head and neck after cancer of the oral cavity. The primary causes of death in cases of laryngeal cancer are the recurrence of locoregional disease and distant metastasis. Anatomic and tissue alterations resulting from surgery and/or radiotherapy of primary laryngeal tumors can make it difficult to determine a locoregional recurrence or residual disease by physical examination or computed tomography (CT)/magnetic resonance imaging (MRI). The majority of studies have shown a high accuracy in the detection of local and regional recurrence of head and neck cancer after different treatment modalities, using fluorodeoxyglucose (FDG)-positron emission tomography (PET)/CT.

Aim: To determine the diagnostic accuracy of PET/CT in patients with suspicion of locoregional recurrence from laryngeal carcinoma after surgery with or without adjuvant radiotherapy.

Materials and methods: This was a retrospective study. Forty-five patients who previously underwent surgical treatment with or without adjuvant radiotherapy for primary laryngeal squamous cell carcinoma and who underwent examination using FDG-PET/CT imaging after clinical and instrumental (CT/MRI) suspicion of locoregional recurrence ( $\mathrm{T}$ or $\mathrm{N}$ ) were recruited. Results: Overall specificity, sensitivity, and accuracy of PET/CT were found to be $88 \%, 100 \%$, and $93.3 \%$, respectively. With respect to the suspected cases of recurrence in the primary site, sensitivity, specificity, and accuracy of PET/CT were found to be $100 \%, 87.5 \%$, and $91.6 \%$, respectively. In patients with suspected metastatic neck disease, PET/CT revealed a sensitivity, specificity, and accuracy of $100 \%, 90 \%$, and $95.4 \%$, respectively.

Conclusion: According to the results of this study, PET/CT imaging in laryngeal tumors is a useful tool in case of suspected locoregional recurrence where conventional imaging (CT and MRI) is unable to resolve the diagnostic doubt.

Keywords: laryngeal cancer, PET/CT imaging, laryngeal cancer recurrence, laryngeal cancer $\mathrm{PET} / \mathrm{CT}$ imaging

\section{Introduction}

Laryngeal cancer is the second most common cancer of the head and neck, after cancer of the oral cavity; it is the sixth most common cancer worldwide and constitutes $28 \%$ of the cancers of the aerodigestive tract. The majority of these tumors are squamous cell carcinoma. ${ }^{1}$

In the last 30 years, progress in the treatment of head and neck cancer has improved the quality of life of these patients through the use of more innovative surgical techniques and multimodal treatments aimed at organ preservation. ${ }^{2,3}$ However, the survival of patients, especially with advanced disease, has not improved. The primary causes 
of death are represented by the recurrence of locoregional disease and distant metastasis. ${ }^{4}$

Anatomic and tissue alterations resulting from surgery with or without radiotherapy in laryngeal tumors can make it difficult to determine locoregional recurrence or residual disease with physical examination or computed tomography (CT)/magnetic resonance imaging (MRI). ${ }^{5,6}$ Moreover, after primary therapy, it is important to accurately identify early recurrence, as salvage treatment is less successful once the disease reaches the advanced stages. ${ }^{7,8}$

Positron emission tomography (PET) has recently demonstrated high reliability in pretreatment staging, radiotherapy planning, and evaluation of the response to chemo/ radiotherapy treatment of head and neck tumors. ${ }^{9,10}$ PET/CT imaging is based on tumor glucose metabolism and serves as a marker to detect metabolic activity of the tumor. Combining PET with CT provides useful functional and anatomical information. ${ }^{11}$

The majority of the studies have shown a high accuracy in the detection of local and regional recurrence of head and neck cancer after different treatment modalities using fluorodeoxyglucose (FDG)-PET/CT. ${ }^{8,12,13}$ However, to our knowledge, studies that consider specific locations and specific treatments are limited.

Different modalities of treatment for different sites may yield different clinical problems and difficulties in detecting recurrent disease. In laryngeal tumors, studies until now were chiefly concerned with the diagnostic accuracy of PET/ $\mathrm{CT}$ and its use in staging pretreatment; however, there are a limited number of studies with respect to the use of PET/ $\mathrm{CT}$ in the evaluation of the response after treatment, in particular, chemo/radiotherapy or radiotherapy. ${ }^{14,15}$ The small number of studies about PET use in the diagnosis of recurrent laryngeal tumors can be attributed to the fact that PET is not included in the guidelines regarding imaging examinations to be performed during follow-up. Indeed, there is no widely accepted protocol for the use of PET/CT in the surveillance of head and neck cancer. ${ }^{16}$

Therefore, in this study, we aimed to determine the diagnostic accuracy of PET/CT in patients with suspicion of locoregional recurrence from laryngeal carcinoma after surgery.

\section{Materials and methods \\ Patients}

This is a retrospective study conducted on patients who underwent surgical treatment with or without adjuvant radiotherapy for laryngeal squamous cell carcinoma and who underwent
PET/CT imaging from January 2010 to January 2012. The study protocol was approved by the Institutional Review Board (IRB) of "Magna Graecia" University of Catanzaro and Cannizzaro Hospital of Catania, Italy. Patient's informed consent was not required by both IRBs, but all patients provided written informed consent for their medical information to be used anonymously for research and educational purposes, and for their images to be published.

Patients who underwent FDG-PET/CT imaging during their follow-up visit due to clinical and instrumental (CT/ MRI) suspicion of locoregional recurrence ( $\mathrm{T}$ or $\mathrm{N}$ ) and those with at least 6 months of follow-up after PET/CT imaging were included in this study.

The disease stage in all patients was clinically assessed according to the seventh edition of the TNM classification established by the American Joint Committee on Cancer (AJCC). ${ }^{17}$

All patients, after the clinical evaluation performed at about 1 month after the surgery, were regularly followed for every 3 months by clinical examination, fiber endoscopy, and ultrasonography, and CT every 6 months. A true-positive finding was confirmed when the PET/CT image was reputed as a malignant lesion and the patient was found to have a local recurrence or a neck metastatic disease based on bioptical or surgical and histopathological results.

False-positive findings occurred when the PET/CT image was positive as malignant disease, but the patient was found to have negative histopathology.

A true-negative finding occurred when PET/CT was reputed as negative for malignant disease and the patient showed negative histopathology or stable clinical outcome over a minimum follow-up period of 6 months. A falsenegative finding occurred when the PET/CT image was negative for malignant disease and the patient had persistent and progressive signs of disease over a minimum follow-up period of 6 months.

The sensitivity, specificity, and accuracy of PET/CT imaging method for assessing recurrence were evaluated. Statistical analysis was performed using MedCalc software.

\section{PET/CT procedure}

PET/CT measurements without contrast were performed on a time-of-flight Discovery 690 scanner (General Electric Medical Systems). Patients who fasted for $6 \mathrm{~h}$ before PET/ CT studies were evaluated, and only patients with a serum glucose level less than $200 \mathrm{mg} / \mathrm{dL}$ were intravenously injected with ${ }^{18} \mathrm{~F}-\mathrm{FDG}$ (37 MBq/10 kg body weight), in accordance with the guidelines of the European Association of Nuclear Medicine (EANM). ${ }^{18}$ The weight of each patient 
was measured on the same scale on the day of the PET/CT imaging. For each patient, the preinjection and residual activities were assayed in a dose calibrator to determine the actual administered activity. Static PET/CT acquisition started 50-60 min after radiotracer injection according to the above-cited recommendations. Patients were positioned with their arms raised and were allowed to breathe normally during PET/CT examinations. All studies were performed from the top of the skull to the middle of the thigh, which included a scout scan at $10 \mathrm{~mA}$, a CT scan $(120 \mathrm{kVp}, 50-120 \mathrm{~mA})$, and a 3D whole-body PET scan ( $2 \mathrm{~min} /$ bed position). PET images were reconstructed using the ordered subset expectation maximization (OSEM) algorithm optimized for time-of-flight and with corrections for random, scatter, and attenuation. For each bed position, the $\mathrm{CT}$ volume consisted of $512 \times 512$ voxels of $1.36 \times 1.36 \times 3.75 \mathrm{~mm}^{3}$ size, whereas the PET image volume consisted of $256 \times 256$ voxels of $2.73 \times 2.73 \times 3.27 \mathrm{~mm}^{3}$ size

PET, CT, and fused PET/CT images were reviewed in axial, coronal, and sagittal planes. To view the image, PET and CT datasets were transferred to an independent computer workstation by Digital Imaging and Communications in Medicine (DICOM) transfer. Semiquantitative and volumetric analyses of the primary tumors were performed by 2 physicians with expertise in nuclear medicine using the volume viewer software on a GE Advantage Workstation 4.6 (GE Healthcare, Milwaukee, WI, USA).

All examinations were reviewed by 2 blinded specialists in head and neck nuclear medicine (MI and ST) with 10 years of experience in reading whole-body images of patients with head and neck cancer. Specific standardized uptake value thresholds were not applied in this study because of the lack of correlation between standard uptake value (SUV) cutoff and diagnostic accuracy of PET/CT. ${ }^{19}$

$\mathrm{PET} / \mathrm{CT}$ images were reported as positive or concerning malignant neoplasm, using a visual and semiquantitative analysis, if the findings met the following criteria: 1) soft tissue or lymph nodes with subjectively increased FDG avidity that could not be attributed to normal or altered physiologic uptake; 2) nodes were also considered malignant if their longest axial diameter was $>15 \mathrm{~mm}$ for levels I and II or $>10 \mathrm{~mm}$ for levels III-V, if they appeared spherical (rather than flat or bean-like) in shape or showed rim enhancement with central necrosis or cystic degeneration, and if they were abnormally grouped..$^{20,21}$ If there were multiple lymph nodes at a specific level, the node suspected to have the highest malignant potential on $\mathrm{CT}$ or showing the highest SUVmax on PET/CT was assessed; 3) pulmonary nodules judged as suspicious based on CT criteria or increased FDG uptake; and 4) distant masses with increased FDG avidity or CT features that were suggestive of malignant neoplasm. We have not made the inter-observer and intra-observer variability since the examiners followed the same as previously defined evaluation criteria; however, this is a limitation of the study.

\section{Results}

Forty-five from a total of 78 patients were included in this study: 42 men and 3 women, with a mean \pm standard deviation (SD) age of $64 \pm 10.2$ years (range: $42-79$ years). The remaining 33 patients were excluded because they did not meet inclusion criteria.

Ten patients had squamous cell carcinoma of the supraglottic region, 18 of the glottic site, and 17 of the transglottic site. According to the TNM classification, 2 were classified as T1b, 14 as T2, 17 as T3, and 12 as T4, whereas according to classification 27 were classified as N0,12 were N1, and 6 were N2.

Sixteen out of 45 cases were of supracricoid laryngectomy (SCL), 4 of supraglottic laryngectomy (SGL), 24 of total laryngectomy (TL), and 1 of tracheoioidopexy (THP). Twenty-four of the 45 patients (53.3\%) underwent unilateral (12 cases) or bilateral (12 cases) neck dissection.

Table 1 shows the demographic and clinicopathological patient data.

The average interval time between the end of treatment and PET/CT imaging execution was $9 \pm 6.2$ months (range: 6-27 months).

In 24 out of 45 cases (53.3\%), the survey was performed for a suspected recurrence of $\mathrm{T}$, whereas in 21 out of 45 cases (46.6\%), the survey was performed for suspicion of $\mathrm{N}$.

In 24 patients with suspected recurrence of $\mathrm{T}$, there were 12 cases of SCL, 2 cases of SGL, 9 cases of TL, and 1 case of THP.

In 10 of these 24 patients, PET/CT examination was positive, and then the cases were submitted for biopsy. Histological examination of the bioptical material revealed the presence of squamous cell carcinoma in 8 cases and granulation tissue in 2 cases ( $1 \mathrm{TL}$ and $1 \mathrm{SCL}$ ). The remaining 14 patients who were negative at $\mathrm{PET} / \mathrm{CT}$ where negative to biopsy and did not currently show further signs of recurrence at the primary site. In Figure 1, we report a case of T recurrence after a SGL that involved the right true and false vocal cord.

In 21 patients with suspected metastatic neck disease, 15 were previously submitted for bilateral ( 8 cases) or unilateral ( 7 cases) neck dissection and 5 of them had undergone 
Table I Demographic and clinicopathological patient data

\begin{tabular}{|c|c|}
\hline $\begin{array}{l}\text { Age } \\
\text { Median } \pm \text { SD } \\
\text { Range } \\
\end{array}$ & $\begin{array}{l}\text { Total patients } \mathbf{N}=\mathbf{4 5} \\
64 \pm 10.2 \\
42-79\end{array}$ \\
\hline \multicolumn{2}{|l|}{ Site } \\
\hline Supraglottic & 10 \\
\hline Glottic & 18 \\
\hline Transglottic & 17 \\
\hline \multicolumn{2}{|l|}{ T classification } \\
\hline TIb & 2 \\
\hline $\mathrm{T} 2$ & 14 \\
\hline T3 & 17 \\
\hline $\mathrm{T} 4$ & 12 \\
\hline \multicolumn{2}{|l|}{$\overline{\mathbf{N}}$ classification } \\
\hline 0 & 27 \\
\hline I & 12 \\
\hline 2 & 6 \\
\hline \multicolumn{2}{|l|}{$\bar{T}$ treatment } \\
\hline $\mathrm{SCL}$ & 16 \\
\hline SGL & 4 \\
\hline TL & 24 \\
\hline THP & I \\
\hline \multicolumn{2}{|l|}{$\mathbf{N}$ treatment } \\
\hline None & 21 \\
\hline SCL monolateral & 12 \\
\hline SCL bilateral & 12 \\
\hline \multicolumn{2}{|l|}{ Radiotherapy } \\
\hline Yes & 13 \\
\hline No & 32 \\
\hline
\end{tabular}

Abbreviations: SD, standard deviation; SCL, supracricoid laryngectomy; SGL, supraglottic laryngectomy; TL, total laryngectomy; THP, tracheoioidopexy.
A

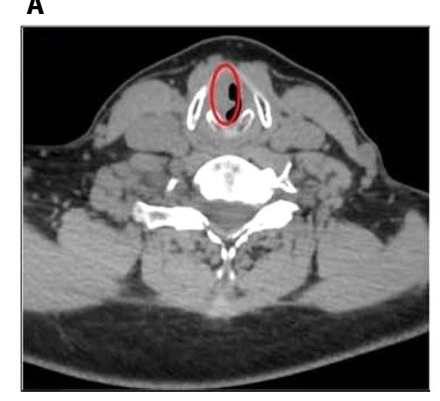

B

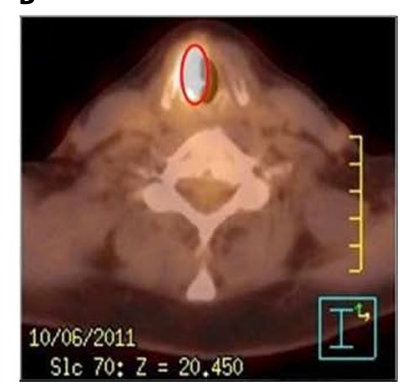

Figure I Whole-body fluorodeoxyglucose (FDG)-positron emission tomography (PET)/computed tomography (CT) images showing focal tracer's uptake (maximum standard uptake value 10,47) located in the right true and false vocal cord. (A) CT image, (B) PET/CT image. The red oval in imaging indicates the lesion.

adjuvant radiotherapy. In 12 of these 21 patients, PET/CT revealed signs of disease in the neck and were submitted for neck dissection (10 cases) and lymphoadenectomy ( 2 cases).

Histological examination of the surgical material in 11 of the 12 cases confirmed the results of PET/CT, which was lymph node metastasis of squamous cell carcinoma, and 1 case was negative. Nine patients with negative PET/CT were followed for more than 6 months and showed no signs of progression of neck disease. All patients had a histological diagnosis within 10 days of the PET/CT imaging execution.

In all the 45 patients, $\mathrm{PET} / \mathrm{CT}$ revealed a specificity, sensitivity, and accuracy of $88 \%, 100 \%$, and $93.3 \%$, respectively.

Analyzing the cases of suspected recurrence in primary site separately, PET/CT revealed a sensitivity, specificity, and accuracy of $100 \%, 87.5 \%$, and $91.6 \%$, respectively.

In patients with suspected metastatic neck disease, PET/ CT revealed a sensitivity, specificity, and accuracy of $100 \%$, $90 \%$, and $95.4 \%$, respectively (Table 2 ). However, we must consider that, in these cases, the suspected metastatic recurrence in 15 of the 21 cases involved a side or a neck not previously treated.

\section{Discussion}

In literature, there are studies regarding the use of PET/ CT imaging in laryngeal tumors, which primarily concern its utility in pretreatment staging for the determination of laterocervical lymph nodes to optimize the indication of neck dissection. ${ }^{22}$ There are also a limited number of studies about its use for the evaluation of radiotherapy response or disease recurrence after radiotherapy or chemoradiation treatment. $^{23}$

To our knowledge, this is the first study to assess the reliability of PET/CT in the diagnosis of suspected local recurrence or lymph node in patients with laryngeal cancer previously treated with open surgery. In these patients, the anatomical and structural disturbance is greater than those who underwent conservative endoscopic surgery or radiotherapy or chemo/radiotherapy. The presence of fibrosis and edema and the asymmetry of the soft tissues and residual anatomic structures make it difficult to diagnose the recurrence. In our study, PET/CT revealed a sensitivity, specificity, and accuracy of $100 \%, 88 \%$, and $93.3 \%$, respectively.

Oe et al, in a study of 28 patients who underwent different treatment modalities for laryngeal cancer, reported a sensitivity, specificity, and accuracy in the diagnosis of locoregional recurrence of $93.75 \%, 91.67 \%$, and $92.86 \%$, respectively. ${ }^{24}$ Robin et al, in a study of patients with laryngeal cancer undergoing different treatment modalities

Table 2 Value of positron emission tomography (PET)-computed tomography (CT) in the diagnosis of primary or nodal laryngeal cancer recurrence

\begin{tabular}{llll}
\hline Site of recurrence & Sensitivity (\%) & Specificity (\%) & Accuracy (\%) \\
\hline Primary tumor & $8 / 8(100)$ & $14 / 16(87.5)$ & $22 / 24(91.6)$ \\
Nodal & $1 \mathrm{I} / \mathrm{II}(100)$ & $10 / 11(90)$ & $2 \mathrm{I} / 22(95.4)$ \\
\hline
\end{tabular}


in which PET/CT was performed 6 months apart, reported a sensitivity, specificity, and accuracy of $96 \%, 87 \%$, and $89 \%$, respectively. ${ }^{25}$ Recently, Citil et al reported PET/CT sensitivity, specificity, and accuracy equal to $100 \%$ in a study of 20 patients with laryngeal cancer, wherein 10 of these patients had not yet been subjected to any treatment. ${ }^{26}$

These studies confirm the high reliability of PET/CT imaging for the diagnosis of locoregional recurrence in laryngeal carcinomas. However, these studies capture some differences that represent the dishomogeneity of the sample of patients examined in these studies. If we consider the results obtained in our study separately, depending on the site of suspected recurrence, we find that, in the assessment of local recurrence $(\mathrm{T})$, sensitivity, specificity, and accuracy were $100 \%, 87.5 \%$, and $91.5 \%$, respectively. However, in patients with suspected metastatic disease (N), PET/CT sensitivity, specificity, and accuracy were $100 \%, 90 \%$, and $95.4 \%$, respectively. It is noteworthy that we have not performed the inter-observer and intra-observer variability, and this is a limitation of this study.

Previous studies regarding the use of PET/CT for laryngeal cancer staging or detection of recurrence of metastatic disease reported very different results in terms of sensitivity, specificity, and accuracy. ${ }^{26-28}$ The increased reliability of PET/ $\mathrm{CT}$ in the evaluation of metastatic nodal disease of the neck with respect to local recurrence can be attributed to the fact that, in $71.4 \%$ of cases, the site of lymph node metastases in the neck has not previously been subjected to dissection.

These data also confirm that in patients undergoing open surgery, diagnosis is highly constrained by tissue typing and anatomical abnormalities following treatment.

The introduction of PET/CT imaging in the guidelines for the follow-up of patients with laryngeal cancer is still under debate, because it is not yet proven that its use in asymptomatic patients gives an advantage in terms of survival. ${ }^{29}$

However, PET/CT outlines its increasingly important role in patients who show signs of relapse during follow-up.

\section{Conclusion}

According to our results, PET/CT imaging in patients with laryngeal tumors was found to be a useful tool in cases of suspected locoregional recurrence where conventional imaging (CT and MRI) was unable to resolve the diagnostic doubt.

\section{Disclosure}

The authors report no conflicts of interest in this work.

\section{References}

1. Ferlay J, Shin HR, Bray F, Forman D, Mathers C, Parkin DM. Estimates of worldwide burden of cancer in 2008: GLOBOCAN 2008. Int $J$ Cancer. 2010;127(12):2893-2917.

2. Allegra E, Franco T, Trapasso S, Domanico R, La Boria A, Garozzo A. Modified supracricoid laryngectomy: oncological and functional outcomes in the elderly. Clin Interv Aging. 2012;7:475-480.

3. Garozzo A, Allegra E, La Boria A, Lombardo N. Modified supracricoid laryngectomy. Otolaryngol Head Neck Surg. 2010;142(1):137-139.

4. Spector JG, Sessions DG, Haughey BH, et al. Delayed regional metastases, distant metastases, and second primary malignancies in squamous cell carcinomas of the larynx and hypopharynx. Laryngoscope. 2001;111(6):1079-1087.

5. Lell M, Baum U, Greess $\mathrm{H}$, et al. Head and neck tumors: imaging recurrent tumor and post-therapeutic changes with CT and MRI. Eur J Radiol. 2000;33(3):239-247.

6. de Bree R, van der Putten L, Brouwer J, Castelijns JA, Hoekstra OS, Leemans CR. Detection of locoregional recurrent head and neck cancer after (chemo)radiotherapy using modern imaging. Oral Oncol. 2009;45(4-5):386-393.

7. Goodwin WJ Jr. Salvage surgery for patients with recurrent squamous cell carcinoma of the upper aerodigestive tract: when do the ends justify the means? Laryngoscope. 2000;110(3 Pt 2 Suppl 93):1-18.

8. Wong LY, Wei WI, Lam LK, Yuen AP. Salvage of recurrent head and neck squamous cell carcinoma after primary curative surgery. Head Neck. 2003;25(11):953-959.

9. Purohit BS, Ailianou A, Dulguerov N, Becker CD, Ratib O, Becker M. FDG-PET/CT pitfalls in oncological head and neck imaging. Insights Imaging. 2014;5(5):585-602.

10. Tantiwongkosi B, Yu F, Kanard A, Miller FR. Role of (18)F-FDG PET/ $\mathrm{CT}$ in pre and post treatment evaluation in head and neck carcinoma. World J Radiol. 2014;6(5):177-191.

11. Schöder H, Yeung HW, Gonen M, Kraus D, Larson SM. Head and neck cancer: clinical usefulness and accuracy of PET/CT image fusion. Radiology. 2004;231(1):65-72.

12. McCollum AD, Burrell SC, Haddad RI, et al. Positron emission tomography with $18 \mathrm{~F}$-fluorodeoxyglucose to predict pathologic response after induction chemotherapy and definitive chemoradiotherapy in head and neck cancer. Head Neck. 2004;26(10):890-896.

13. Nam SY, Lee SW, Im KC, et al. Early evaluation of the response to radiotherapy of patients with squamous cell carcinoma of the head and neck using 18FDG-PET. Oral Oncol. 2005;41(4):390-395.

14. Brouwer J, Hooft L, Hoekstra OS, et al. Systematic review: accuracy of imaging tests in the diagnosis of recurrent laryngeal carcinoma after radiotherapy. Head Neck. 200830(7):889-897.

15. van der Putten L, Hoekstra OS, de Bree R, et al. 2-Deoxy-2[F-18] FDG-PET for detection of recurrent laryngeal carcinoma after radiotherapy: interobserver variability in reporting. Mol Imaging Biol. 2008;10(5):294-303.

16. Associazione Italiana di Oncologia Cervico-Cefalica (AIOCC). Linee guida multidisciplinari AIOCC AIRO AIOM. Tumori della testa e collo algoritmi diagnostico terapeutici. [Italian Association of CervicalCephalic Oncology (AIOCC). Lines Multidisciplinary AIOCC AIRO AIOM guide. Head and Neck Tumors Therapeutic diagnostic algorithms.] Version 2 (April) 2012. Italian.

17. Sobin LH, Gospodarowicz MK, Wittekind Ch. TNM Classification of Malignant Tumours. 7th ed. Oxford, UK: Wiley-Blackwell; 2009.

18. Boellaard R, Delgado-Bolton R, Oyen WJ, et al; European Association of Nuclear Medicine (EANM). FDG PET/CT: EANM procedure guidelines for tumour imaging: version 2.0. Eur J Nucl Med Mol Imaging. 2015;42(2):328-354.

19. Tan A, Adelstein DJ, Rybicki LA, et al. Ability of positron emission tomography to detect residual neck node disease in patients with head and neck squamous cell carcinoma after definitive chemoradiotherapy. Arch Otolaryngol Head Neck Surg. 2007;133(5):435-440. 
20. Sakai O, Curtin HD, Romo LV, Som PM. Lymph node pathology. Benign proliferative, lymphoma, and metastatic disease. Radiol Clin North Am. 2000;38(5):979-998, x.

21. Som PM, Curtin HD, Mancuso AA. Imaging-based nodal classification for evaluation of neck metastatic adenopathy. AJR Am J Roentgenol. 2000;174(3):837-844.

22. Roh JL, Park JP, Kim JS, et al. 18F fluorodeoxyglucose PET/CT in head and neck squamous cell carcinoma with negative neck palpation findings: a prospective study. Radiology. 2014;271(1):153-161.

23. de Bree R, van der Putten L, van Tinteren H, et al. Effectiveness of an (18)F-FDG-PET based strategy to optimize the diagnostic trajectory of suspected recurrent laryngeal carcinoma after radiotherapy: the RELAPS multicenter randomized trial. Radiother Oncol. 2016; 118(2):251-256.

24. Oe A, Kawabe J, Torii K, et al. Detection of local residual tumor after laryngeal cancer treatment using FDG-PET. Ann Nucl Med. 2007;21(1): 9-13.
25. Robin P, Abgral R, Valette G, et al. Diagnostic performance of FDG $\mathrm{PET} / \mathrm{CT}$ to detect subclinical HNSCC recurrence 6 months after the end of treatment. Eur J Nucl Med Mol Imaging. 2015;42(1): $72-78$.

26. Citil S, Dogan S, Atilgan HI, et al. Comparison of dynamic contrastenhanced MRI and PET/CT in the evaluation of laryngeal cancer after inadequate CT results. Pol J Radiol. 2015;80:428-432.

27. Gilbert MR, Branstetter BF 4th, Kim S. Utility of positron-emission tomography/computed tomography imaging in the management of the neck in recurrent laryngeal cancer. Laryngoscope. 2012;122(4):821-825.

28. Yamazaki Y, Saitoh M, Notani K, et al. Assessment of cervical lymph node metastases using FDG-PET in patients with head and neck cancer. Ann Nucl Med. 2008;22(3):177-184.

29. Ho AS, Tsao GJ, Chen FW, et al. Impact of positron emission tomography/computed tomography surveillance at 12 and 24 months for detecting head and neck cancer recurrence. Cancer. 2013;119(7): 1349-1356.

\section{Publish your work in this journal}

Reports in Medical Imaging is an international, peer-reviewed, open access journal publishing original research, reports, reviews and commentaries on all areas of medical imaging. The manuscript management system is completely online and includes a very quick and fair peer-review system, which is all easy to use.

\section{Dovepress}

Visit http://www.dovepress.com/testimonials.php to read real quotes from published authors. 